\title{
SIENA AND ITALIAN LYMPHOLOGY: FROM MASCAGNI TO COMPARINI....TO TOSATTI. \\ A “TRANSLATIONAL" OVERVIEW FROM THE ORIGINS UNTIL TODAY, \\ FROM SIENA TO GENOA, IN ITALY.
}

\section{Campisi C. ${ }^{1}$, Boccardo F. ${ }^{1}$, Acconcia A. ${ }^{2}$, Campisi Caterina ${ }^{1}$, Campisi C.C. ${ }^{3}$}

\author{
${ }^{1}$ Dpt.of Surgical Sciences and Integrated Diagnostics (DISC), IRCCS (Institute of Research and Care at \\ Scientific Character), University Hospital San Martino -IST, Genoa, Italy \\ "2"Emeritus" Head Physician of Urology, Siena and Roma Hospital \\ ${ }^{3}$ Dpt.of Health Sciences (DISSAL), IRCCS (Institute of Research and Care at Scientific Character), Uni- \\ versity Hospital San Martino -IST, Genoa, Italy
}

\section{Historic, SCIENTIFIC ANd IDEOLOGicAl BACKGROUND}

On April 19, 2010, the dream I had at the beginning of my career as University Surgeon became true (C.C.): namely, to have the opportunity to hold a lecture at the Accademia dei Fisiocritici in Siena, visit the Mausoleum dedicated to Paolo Mascagni (Fig.1), live again the "intellectual ecstasy" my beloved and unforgettable Master - Prof. Egidio Tosatti - enjoyed back in the '50s. As Director in those years of the Surgery Department at the University of Siena, working at the Pellegrinaio degli Spedali of Santa Maria della Scala, Prof. Tosatti had acquired "direct" knowledge of the anatomic studies conducted by Mascagni, and started the wonderful journey of modern Italian Lymphology. His work was to be continued and further developed and consolidated in Genoa, from the '60s until today, also through the diligence and hard work of his Pupils.

Therefore, I heartily thank the President of the Accademia, Prof. Sara Ferri, and the President of this Conference in Siena, Prof. Mario Comporti and Dr. Chiara Bratto, in charge of communications and cultural events at the Accademia. In particular, I wish to thank Professor Angelo Acconcia, Prof. Tosatti's first Pupil, in Siena, who followed up and developed the research work that has paved the way to the Modern Age of Lymphatic Surgery.

Hence, in this sort of "flight of fancy", from Mascagni, to Comparini and Tosatti, we get to my visit to the Accademia dei Fisiocritici, gener- ously "prepared" by Prof. Angelo Acconcia and "guided" by none less than the President of the Accademia herself, Professor Sara Ferri. Just imagine the emotion and commotion!...

Now, back to the recollections and historic memories of my youth years, as an intrepid pupil at the side of the great Professor Tosatti: when I graduated he gave me as a present the Book of Proceedings (Fascicolo degli Atti) of the Accademia, curated by Vincenzo Mazzi, dedicated to "Gli Anatomici Senesi del '700" and to Giovanni Battista Bianchi, Janus Plancus, and describing his relations with the University and the City of Siena (Fig.2).

Professor Tosatti, when presenting the book to me, told me - and also wrote in the title page of the book - that he wished to be remembered as the President of the Medical Section of the Accademia dei Fisiocritici!

Of this Fascicolo, which I have had the great pleasure to read again on this occasion, I would like to mention also the learned Introduction by Prof. Mauro Barni, then Rector of the University of Siena, which confirms

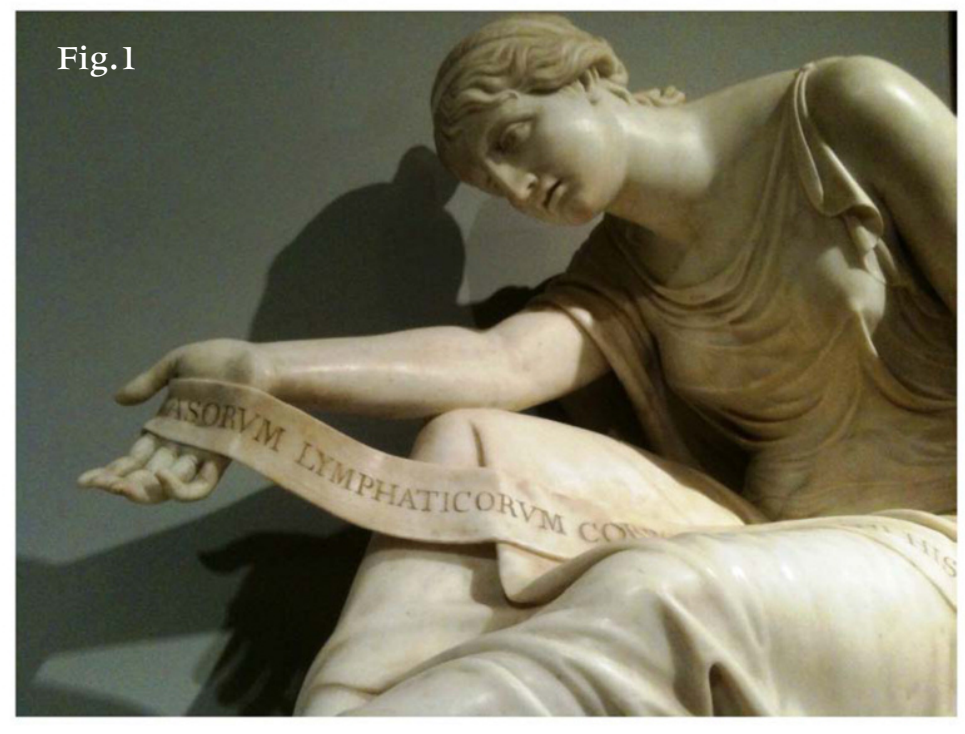
once again the historic relations between the Accademia, the University and the City of Siena.

Since its foundation in 1691 by Pirro Maria Gabrielli, a Siena-born patrician and Professor of Theoretical Medicine and Botany, the Accademia undoubtedly enjoyed its golden age, with the spread of scientific culture, mostly during the presidency of the Anatomist Paolo Mascagni, from 1798 to

Operative Unit of General Surgery and Lymphatic Surgery, Dpt.of Surgical Sciences and Integrated Diagnostics (DISC), IRCCS (Institute of Research and Care at Scientific Character), University Hospital San Martino -IST, National Institute for Cancer Research, Genoa,Italy. Tel. (+39) 0105600-125/-861

E-mail: campisi@unige.it 


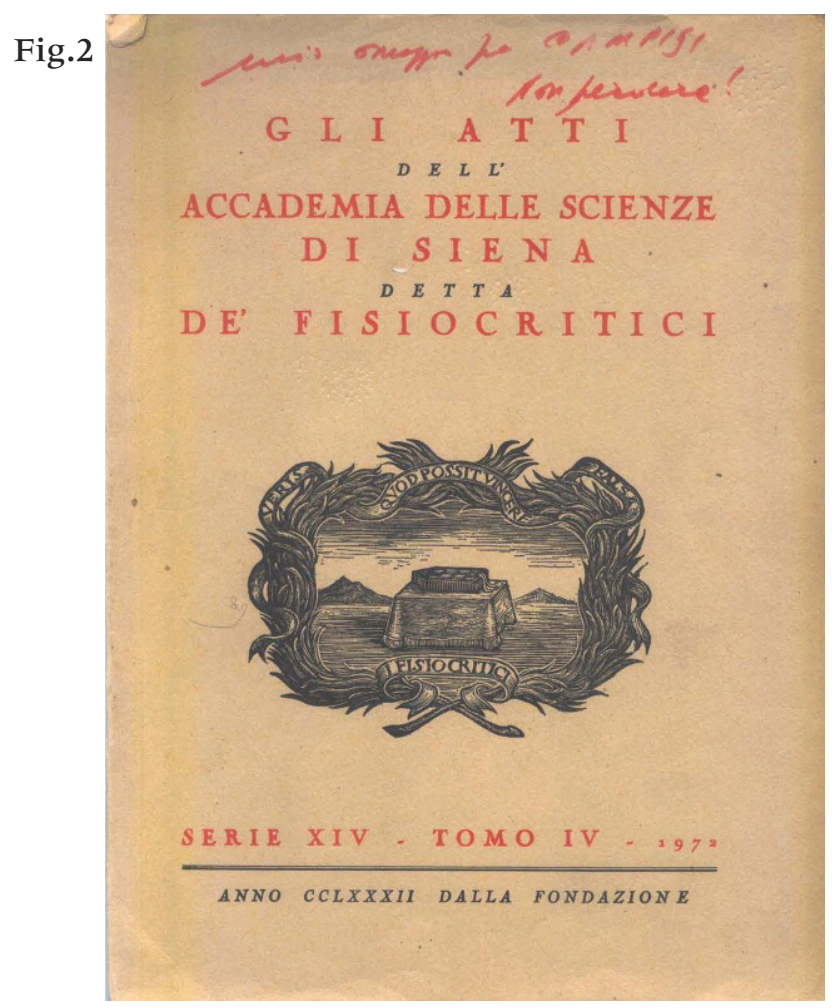

1815. In 1891, the Accademia even hosted the 14th Congress of the Italian Association of Medicine.

Paolo Mascagni, born in Pomarance, near Volterra, in 1755, graduated in Philosophy and Medicine in Siena, in 1778 ("cum optimo responso... et optimo voto"). Only two months after his graduation, Grand Duke Leopold II appointed him Lecturer of Human Anatomy, to replace his renowned Master, Pietro Tabarrani, who was sick.

In 1787, his investigations on "absorbing serous or lymphatic vessels" brought him international renown, with the publication of his work entitled "Vasorum Lymphaticorum Corporis Humani Historia et Ichonographia", as well as his posthumous work entitled "Grande Anatomia".

Mascagni's Anatomic Tables - and the particularly famous full-scale ones- that we may admire in the rooms of the Accademia dedicated to him, are the first and still unequalled model of a Systematic Study of Lymphatic Vessels, with an unparalleled wealth of detailed and minute information about the "Lymphatic System".

After his election as President of the Accademia dei Fisiocritici, in 1798, due to some political difficulties, Mascagni had to leave Siena and moved to Pisa, where he was appointed Full Professor of Human Anatomy in 1801. Then, in 1803, he was called to Florence.

Still very active as Scientist and Master, during a resting period in his estate in Castelletto, near his birthplace of Pomarance, he developed a malignant fever and died on October 19, 1815 at only sixty years of age. However, the "fertilizing seed" of the Modern Lymphologic Science had been spread! About 150 years later... Professor Egidio Tosatti, Director of the Surgery Department of Siena, stimulated by his contacts with the Accademia dei Fisiocritici, - where, a few years later, he would be appointed President of the Medical Sec- tion (1959-1965) - and, above all, intrigued by the studies conducted by the Great Master Mascagni, had the idea of getting some anatomic "samples" X-rayed. Both deep and superficial lymphatic vessels in these samples had been prepared by Mascagni, with an injection of mercury, by means of a glass microneedle cannula obtained with "microsurgical" accuracy with a Bunsen burner. Mercury was injected into the foot lymphatic vessels while the surgeon had to keep his hand quite firm ("cubito bene firmato").

It was the dawn of Lymphatic Microsurgery, marking the beginning of a new age of studies and research of the Lymphatic System, which would lead to the definition and, later, the "standardization of Lymphographic Diagnostics".

In those years, the contribution of Professor Angelo Acconcia was fundamental, who, meanwhile, had been sent to London by Professor Tosatti to personally follow the innovative clinical and research work conducted by the great Professor J. B. Kinmonth (1955), 1 who had actually been preceded by another great Professor, Marceau Servelle (1944), 2 pupil of another great Master, René Leriche.

Hence, from Mascagni, through Tosatti and the standardization of lymphographic techniques, for the study of both superficial and deep lymphatic vessels, the foundation was laid to modern Lymphatic Surgery and Microsurgery.

The magic age of this Siena-centred "season" in the study of Lymphatic vessels was also enlightened in those years by yet another great Anatomist, also from Siena: Prof. Leonetto Comparini.

His studies make up the basis of our current knowledge about hydrodynamic and anti-gravitational functions of lymph nodes correlated with the leiomuscular component of the lymph node capsule. With its slow, alternated contraction and release, the lymph node is like a small "heart" ), synchronized with the systolicdiastolic movements of "lymphangions", the anatomicfunctional unit of each lymphatic collector. It is also synchronized with the alternated rhythm of the microhistoangic unit, which is synchronous with the opening and closing of endothelial intercellular desmosomal junctions of initial lymphatics (dead-end lymphatics). These are the mechanisms underlying lymphogenesis, governed by Laplace's law and typical of the 'absorbing' and 'carrying' function of lymphatic vessels, in a centripetal mode, from the periphery (interstitial space, initial lymphatics, and pre-collectors) to the centre (lymphatic vessels).

The "Gravitational Assumption" proposed by Prof. Tosatti back in 1967 for the Pathogenesis of Chronic Lymphedemas and the related Classification and Surgical Treatment, featuring ligation of ectatic, dysplastic, and insufficient vessels in case of Gravitational Lymphedemas is based on this knowledge.

Hence, the initially symptomatic, resective, and debulking surgical treatment of Lymphedemas became physiopathologic and functional, targeting the very cause of disease, rather than the result of lymphatic stasis caused by 'gravitational reflux'. 